\title{
Psychological Distress, Felt Stigma, and HIV Prevention in a National Probability Sample of Sexual Minority Men
}

\author{
Evan A. Krueger, PhD, MPH, MSW, Ian W. Holloway, PhD, LCSW, MPH, ${ }^{1}$ \\ Marguerita Lightfoot, $\mathrm{PhD},{ }^{3}$ Andy Lin, $\mathrm{PhD},{ }^{4}$ Phillip L. Hammack, $\mathrm{PhD},{ }^{5}$ and Ilan $\mathrm{H}$. Meyer, $\mathrm{PhD}^{6}$
}

\begin{abstract}
Purpose: We assessed how psychological distress and felt stigma (perceived sexual minority stigma in one's community) are associated with key HIV prevention outcomes in a U.S. national probability sample of sexually active, HIV-negative sexual minority men.

Methods: Using data from the Generations study (2017-2018, N=285), the present study assessed the effects of psychological distress and felt stigma and their interaction on three HIV prevention outcomes: testing for HIV as per Centers for Disease Control and Prevention guidelines (once or more in the past year), use of latex barriers (e.g., condoms), and familiarity with pre-exposure prophylaxis (PrEP).

Results: In main effects models, neither psychological distress nor felt stigma was associated with any of the screening and prevention outcomes. However, the interaction between psychological distress and felt stigma was associated with each outcome. Specifically, at higher levels of felt stigma, greater psychological distress was associated with lower odds of HIV testing (exponentiated coefficient $=0.93$, confidence interval $[95 \% \mathrm{CI}]$ $0.87-1.00$ ), use of latex barriers (exponentiated coefficient $=0.92,95 \%$ CI $0.86-0.99$ ), and familiarity with PrEP (exponentiated coefficient $=0.90,95 \%$ CI $0.82-0.98$ ).

Conclusion: These findings highlight the importance of felt stigma in shaping the association between psychological distress and engagement in HIV screening and prevention and offer important considerations for future HIV prevention research and interventions.
\end{abstract}

Keywords: felt stigma, HIV prevention, MSM, psychological distress

\section{Introduction}

$\mathbf{S}$ EXUAL MINORITY MEN (e.g., gay, bisexual, and other men who have sex with men [MSM]) are disproportionately impacted by HIV. For instance, although gay, bisexual, and other MSM represent $<3 \%$ of the general population of men, ${ }^{1}$ they accounted for $\sim 70 \%$ of new HIV infections in 2017. ${ }^{2}$ Efforts to curb the spread of HIV have relied on early detection, and it is recommended that sexually-active MSM are tested for HIV at least once yearly. ${ }^{3}$ In addition, ample evidence has shown the efficacy of consistent condom use for preventing the transmission of HIV, ${ }^{4}$ and HIV prevention efforts have long promoted condom use among groups most at risk for infection, including sexual minority men. ${ }^{5}$ The emergence of Truvada, a medication originally approved for HIV treatment but more recently (2012) ap- proved for the prevention of HIV transmission (termed "pre-exposure prophylaxis [PrEP]"), changed the landscape of HIV prevention. When taken consistently, PrEP has been shown to reduce the risk for HIV transmission by up to $92 \%{ }^{6}$ However, uptake has been slow across the United States. For instance, despite high levels of familiarity (with $59.8 \%$ being "somewhat" or "very familiar") and largely favorable attitudes toward PrEP (with $68.3 \%$ reporting they were "for [PrEP]",), only $4.1 \%$ of sexually-active HIV-negative sexual minority men reported PrEP use in a recent (2016-2017) national probability sample. ${ }^{7}$ Uptake rates have been similarly low, ranging from $0 \%$ to $12 \%$, across several studies using community-based samples. ${ }^{8-10}$

People experiencing poor mental health (e.g., depression, anxiety, or psychological distress) are at increased risk for acquiring HIV, ${ }^{11,12}$ and sexual minority men experience

\footnotetext{
${ }^{1}$ Department of Preventive Medicine, Keck School of Medicine, University of Southern California, Los Angeles, California, USA.

${ }^{2}$ Department of Social Welfare, Luskin School of Public Affairs, University of California, Los Angeles, Los Angeles, California, USA.

${ }^{3}$ Department of Medicine, University of California, San Francisco, San Francisco, California, USA.

${ }^{4}$ Institute for Digital Research and Education, University of California, Los Angeles, Los Angeles, California, USA.

${ }^{5}$ Department of Psychology, University of California, Santa Cruz, Santa Cruz, California, USA.

${ }^{6}$ The Williams Institute, School of Law, University of California, Los Angeles, Los Angeles, California, USA.
} 
mental health problems at higher rates than heterosexual men. ${ }^{13-16}$ These mental health disparities are driven largely by chronic exposure to stigma and other minority stressors (e.g., discrimination and victimization). ${ }^{17-23}$ Both mental health challenges and exposure to minority stressors have been directly associated with increased engagement in HIV risk behaviors among sexual minority men, including substance use, ${ }^{24-27}$ and decreased engagement in HIV preventive behaviors, including use of condoms and/or PrEP. ${ }^{18,28}$ To adequately address the HIV epidemic and reduce new transmissions, HIV prevention efforts must consider sexual minority men with comorbid mental health problems. ${ }^{29}$

A growing body of research addresses how structural and contextual factors (e.g., presence vs. absence of employment nondiscrimination laws, public attitudes about homosexuality) local to where sexual minority people live affect their health. ${ }^{20,30,31}$ Felt stigma is a minority stress process that refers to expectations of stigma and prejudice related to being gay, bisexual, or MSM, causing stress by requiring chronic vigilance by the gay/bisexual person. ${ }^{32}$ It is assessed as a person's level of consciousness about being stigmatized and devalued by their community. ${ }^{33,34}$ As such, it stands to reason that felt stigma is associated with increased HIV risk. ${ }^{35,36}$ However, the mechanism(s) by which mental health, felt stigma, and engagement in HIV screening and prevention are related are not well understood. For instance, it is possible that felt stigma is associated directly with HIV screening and prevention, but it is also possible that felt stigma modifies (i.e., strengthens) the associations between mental health and HIV screening and prevention. To our knowledge, no studies have assessed this question directly, and the majority of studies related to mental health, stigma, and HIV risk among sexual minority men have been conducted using regional, community, and/or clinic-based samples with limited generalizability. ${ }^{37-40}$ To address these gaps in HIV prevention research, we assessed how psychological distress and felt stigma were associated with three HIV screening and prevention outcomes (HIV testing, condom use, and PrEP knowledge), using a national probability sample of sexually active, HIV-negative sexual minority men.

\section{Methods}

\section{Study design}

Data were from the Generations study, designed to assess the impact of the changing social environment (e.g., laws, policies, and culture) on the health and well-being of lesbian, gay, and bisexual (LGB) individuals. ${ }^{41}$ Three distinct age cohorts (ages 18-25, 34-41, and 52-59 at screening) were selected that represent different historical periods that contextualized the experience of LGB adults in the United States. The Gallup Daily Tracking Survey, a daily national probability sample of 1000 adults ages 18 and older, ${ }^{42}$ was used as initial contact and participants were screened for eligibility in 2016-2017. Participants were eligible if they identified as gay, lesbian, bisexual, queer, or same-gender loving; were not transgender; were ages $18-25,34-41$, or 52-59 at screening; were White, Black, or Latinx; and completed at least a sixth grade education. Eligible respondents were invited to participate in a self-administered survey, either online or by mail. Of the 366,644 respondents who were screened, 3.5\% $(N=12,837)$ identified as LGBT, and of them, $27.5 \%(N=3525)$ met eligibility criteria. A total of 1331 eligible respondents completed the baseline survey. Respondents read an information sheet before beginning the survey and consented by completing the survey. It was determined that no signed consent forms would be collected due to the confidential nature of data collection.

Data from the second wave of data collection (2017-2018; $N=894 ; 67.2 \%$ retention) were used for the present study due to the inclusion of more HIV screening and prevention outcomes at wave 2 than at baseline. In this article, we report on men $(N=285)$ who reported having sex (defined as "any activity you personally define as sexual activity") with another man in the past year and who were HIV negative and, therefore, at risk for HIV. The study was approved by the Institutional Review Boards at University of California, Los Angeles, University of California, San Francisco, University of California, Santa Cruz, University of Texas at Austin, and University College London.

\section{Study variables}

HIV screening and prevention engagement was assessed using three items. HIV testing was assessed using the question, "In the past year, how many times did you get tested for HIV?" (numeric response). Respondents were categorized as meeting Centers for Disease Control and Prevention (CDC) guidelines (testing once or more in the past year) or not (less than once). ${ }^{3}$ Respondents were also asked whether they had used "latex barriers (dental dam, plastic wrap, or condom)" with a male sex partner in the past year. Responses were dichotomous (yes/no). Because too few men reported using PrEP $(N=26)$ to allow stable statistical analyses, we used familiarity with PrEP-a necessary first step toward understanding eligibility, acceptability, and uptake ${ }^{10,43}$ - as an outcome variable assessing PrEP penetration. Familiarity with PrEP was assessed with the question, "Truvada is a pill that HIV-negative people can take to prevent HIV infection. This is called PrEP [or Pre-Exposure Prophylaxis]. How familiar are you with Truvada as PrEP?" (Not at all familiar, somewhat familiar, very familiar). Responses were dichotomized: familiar (somewhat or very familiar) versus not familiar (not at all).

Psychological distress was assessed with Kessler's K-6 measure, a six-item, validated reliable measure of psychological distress (Cronbach's $\alpha$ within the sample $=0.89$ ). ${ }^{44}$ Respondents were asked how often, in the past 30 days, they felt "nervous," "hopeless," "restless or fidgety," "so depressed that nothing could cheer you up," "that everything was an effort," and "worthless." Responses ranged from "none of the time" to "all of the time." Responses to the six items were summed to generate a scale score (range $=$ 0-24). Lower values represent less psychological distress, whereas higher values represent greater distress.

Felt stigma was assessed with the three-item Felt Stigma Scale, which assessed respondents' consciousness about being stigmatized by their community (Cronbach's $\alpha$ within the sample $=0.74) .{ }^{33}$ Scale items were "most people where I live think less of a person who is LGB," "most employers where I live will hire openly LGB people if they are qualified for the job," and "most people where I live would not want someone who is openly LGB to take care of their children." Responses ranged from "strongly disagree" to "strongly 
agree." Items were reverse-coded as necessary, and a scale score was created as a mean of responses to all items within the scale (range $=1-5$ ). Lower values represent less felt stigma, and higher values represent greater felt stigma.

\section{Covariates}

Given well-established associations between several sociodemographic characteristics (e.g., young age and low educational attainment) and HIV risk, ${ }^{2,26}$ the following covariates were included as control variables. For age cohort, respondents were asked "in what year were you born?" A numeric age was calculated by subtracting birth year from the year in which respondents completed the survey (2017 or 2018). Respondents were assigned to one of three age cohorts (19-26, 35-42, 53-60 years). A three-category race/ethnicity variable was calculated based on respondents' reported races ("Which of the following describes your race?") and ethnicities ("Are you of Hispanic, Latino, or Spanish origin - such as Mexican, Puerto Rican, Cuban, or other Spanish origin?") at screening. Respondents were categorized into one of three distinct race/ethnicity categories as White, Black/African American, or Latinx/Hispanic, including in each category additional race/ethnic identities if a respondent reported multiple identities (described in detail elsewhere). ${ }^{41}$ Education was assessed with the question, "What is the highest level of school you have completed or the highest degree you have received?" (high school or less, some college, college completed, more than college completed). Responses were dichotomized (high school or less vs. more than high school). Sexual identity was assessed with the question, "Which of the following best describes your current sexual orientation?"' Response options were "straight/heterosexual," "lesbian,", “gay," "bisexual," "queer," "same-gender loving," and "other." Respondents identifying as heterosexual $(N=8)$ were excluded from the analysis, and the remaining response options were collapsed into one of three categories: gay, bisexual, or other. No respondents identified as lesbian. PrEP use (used as a covariate in one model, see "Data Analysis" section) was assessed with the question, "Are you currently taking Truvada as PrEP?"' (yes/no).

\section{Data analysis}

Descriptive statistics of the sample were first calculated. Multivariable logistic regression analyses then assessed whether psychological distress and felt stigma were associated with each of the three HIV screening and prevention engagement outcomes, adjusting for covariates. PrEP use was included as a covariate in the latex barrier model, but not the HIV testing and PrEP familiarity models, as testing and familiarity are necessary precursors of, and so perfectly predicted, PrEP use. Finally, to assess whether felt stigma modified the association between psychological distress and each outcome, a multiplicative interaction term (i.e., psychological distress $\times$ felt stigma) was added to each multivariate logistic regression model. Psychological distress and felt stigma were mean centered in the interaction models.

Survey weights were developed by Gallup every day after daily recruitment to adjust for nonresponse bias, as described in detail elsewhere. ${ }^{41}$ Briefly, the sampling frame was stratified to ensure that the unweighted sample was proportionate by U.S. Census region and time zone. Then, weights were applied to compensate for disproportionalities in nonresponse and selection probabilities. In addition, because LGBT population characteristics are not included in the U.S. Census, Gallup adjusted weights for the LGBT population based on all of its LGBT samples collected since 2012 (separate LGB data are not available). Weights were applied in all analyses, allowing for generalization to the U.S. population of sexually active, HIV-negative sexual minority men.

\section{Results}

Table 1 presents descriptive statistics for the sample. Over half of the sample $(54.92 \%)$ had been tested for HIV in the past year and approximately one third (36.60\%) used a latex barrier (e.g., a condom or dental dam) with male sex partners in the past year. Although only 26 men $(7.52 \%)$ reported PrEP use, approximately three quarters of the sample reported being "somewhat" or "very" familiar with PrEP (78.73\%). The mean psychological distress score for the sample was 6.68 (standard deviation $[\mathrm{SD}]=4.45$ ), and the mean felt stigma score was $2.40(\mathrm{SD}=0.91)$. About half of the men in the sample $(53.18 \%)$ were in the youngest age cohort, and most $(76.73 \%)$ identified as gay, with an additional $17.13 \%$ identifying as bisexual. Approximately two

Table 1. Sample Characteristics

\begin{tabular}{|c|c|c|}
\hline & $\stackrel{\mathrm{N}}{\text { (weighted \%) }}$ & $\begin{array}{l}\text { Weighted } \\
\text { mean (SD) }\end{array}$ \\
\hline \multicolumn{3}{|l|}{ HIV testing } \\
\hline $\begin{array}{l}\text { Tested once or more } \\
\text { in the past year }\end{array}$ & $143(54.92)$ & - \\
\hline Did not test in the past year & $134(45.08)$ & - \\
\hline \multicolumn{3}{|l|}{ Used latex barrier } \\
\hline Yes & $92(36.60)$ & - \\
\hline No & $193(63.40)$ & - \\
\hline \multicolumn{3}{|l|}{ Familiar with PrEP } \\
\hline Yes & $228(78.73)$ & - \\
\hline No & $56(21.27)$ & - \\
\hline $\begin{array}{l}\text { Psychological distress } \\
\text { (range: 0-24) }\end{array}$ & - & $6.68(4.45)$ \\
\hline Felt stigma (range: $1-5$ ) & - & $2.40(0.91)$ \\
\hline \multicolumn{3}{|l|}{ Age cohort } \\
\hline $19-26$ & $109(53.18)$ & - \\
\hline $35-42$ & $80(27.54)$ & - \\
\hline $53-60$ & $96(19.28)$ & - \\
\hline \multicolumn{3}{|l|}{ Sexual identity } \\
\hline Gay & $228(76.73)$ & - \\
\hline Bisexual & $38(17.13)$ & - \\
\hline Other minority identity & $19(6.14)$ & - \\
\hline \multicolumn{3}{|l|}{ Race/ethnicity } \\
\hline White & $219(64.69)$ & - \\
\hline Black/African American & $21(12.90)$ & - \\
\hline Latinx/Hispanic & $45(22.42)$ & - \\
\hline \multicolumn{3}{|l|}{ Education } \\
\hline High school or less & $48(40.98)$ & - \\
\hline More than high school & $237(59.02)$ & - \\
\hline \multicolumn{3}{|l|}{ PrEP use } \\
\hline Did not use PrEP & $259(92.48)$ & - \\
\hline Used PrEP & $26(7.52)$ & - \\
\hline
\end{tabular}

PrEP, pre-exposure prophylaxis; SD, standard deviation. 
thirds of the men were White $(64.69 \%)$, with the rest identifying as Black/African American (12.90\%) or Latinx/Hispanic $(22.42 \%)$. The majority $(59.02 \%)$ of men reported having more than a high school education.

Table 2 presents multivariable logistic regression analyses, which examined the associations between psychological distress and felt stigma and each HIV screening and prevention engagement outcome, adjusting for covariates. In main effects models, neither psychological distress nor felt stigma was associated with any of the outcomes: HIV testing (psychological distress: adjusted odds ratio $[\mathrm{aOR}]=1.03$, 95\% confidence interval [CI] 0.95-1.11; felt stigma: aOR= $0.71,95 \%$ CI $0.50-1.01$ ), using a latex barrier (psychological distress: $\mathrm{aOR}=0.98$, 95\% CI 0.91-1.07; felt stigma: $\mathrm{aOR}=0.89$, 95\% CI 0.60-1.31), or being familiar with PrEP (psychological distress: OR $=0.99$, 95\% CI 0.90 1.08; felt stigma: $\mathrm{aOR}=0.67,95 \%$ CI $0.44-1.03)$. Unlike in the main effects models, the interaction term was significantly associated with each of the outcomes (HIV testing: exponentiated coefficient $=0.93$, 95\% CI 0.87-1.00; using a latex barrier: exponentiated coefficient $=0.92$, 95\% CI 0.86-0.99; and being familiar with PrEP: exponentiated coefficient $=0.90,95 \%$ CI $0.82-0.98)^{*}$, signifying that as felt stigma increased, greater psychological distress was associated with lower odds of each outcome.

In both main effects and interaction models, older men had lower odds of using latex barriers compared to younger men (main effects: $\mathrm{aOR}=0.22$, 95\% CI 0.09-0.54; interaction: $\mathrm{aOR}=0.22$, 95\% CI 0.09-0.55). Black men (main effects: $\mathrm{aOR}=4.08$, 95\% CI 1.39-11.97; interaction: $\mathrm{aOR}=3.60$, 95\% CI 1.25-10.37) and Latinx men (main effects: aOR = 2.37, 95\% CI 1.00-5.60; interaction: $\mathrm{aOR}=2.66$, 95\% CI 1.14-6.19) had higher odds of using latex barriers, compared to White men. Finally, bisexual men had lower odds of being familiar with PrEP than gay men (main effects: aOR= $0.17,95 \%$ CI $0.06-0.49$; interaction: $\mathrm{aOR}=0.16,95 \% \mathrm{CI}$ 0.06-0.46).

As PrEP use perfectly predicted the HIV testing and PrEP familiarity outcomes, we were unable to control for PrEP use in these models. In subsequent analyses, all three interaction models were run among respondents reporting no PrEP use. Although the interaction term remained significantly associated with use of latex barriers (exponentiated coefficient $=0.92$, 95\% CI 0.85-0.99), the interaction terms lost significance in the HIV testing (exponentiated coefficient $=$ 0.93, 95\% CI 0.86-1.01) and PrEP familiarity models (exponentiated coefficient $=0.91,95 \%$ CI $0.82-1.00)$. As the coefficients and $95 \%$ CIs did not change considerably across models, it is possible that the slight expansion of the $95 \%$ CIs in the restricted models reflects a slight loss in analytic power (PrEP users made up 9\% of our sample). However, it is also possible that these effects were driven by underlying differences between PrEP users and nonusers. Unfortunately, given that PrEP use perfectly predicted the HIV testing and PrEP familiarity outcomes, we could not test this hypothesis.

Figure 1 visually graphs the associations between the interaction (psychological distress $\times$ felt stigma) and each

*The exponentiated coefficient for an interaction term is interpreted as a ratio of two odds ratios. Thus, rather than incorrectly referring to these terms as "odds ratios," we simply refer to them as "exponentiated coefficients" here.
HIV screening and prevention outcome. Each panel displays the association between psychological distress and the predicted probability of the outcome (A: HIV testing, B: use of latex barriers, C: familiarity with PrEP), at three levels of felt stigma (scale range $=1-5 ; 1=$ least stigma, $3=$ median value, $5=$ most stigma). The results show that at the median value of felt stigma (3), higher psychological distress was not significantly associated with any of the HIV prevention outcomes. However, for respondents reporting high levels of stigma (5), higher psychological distress was associated with a lower probability of each outcome. Conversely, at low levels of stigma (1), higher psychological distress was associated with higher probability of each outcome.

\section{Discussion}

Results from this study fill an important gap in our understanding of the psychological and social processes contributing to HIV screening and prevention engagement among sexual minority men. We found that the association between psychological distress and prevention outcomes varied across levels of felt stigma. These findings highlight how despite an average "zero" effect of psychological distress on HIV screening and prevention engagement, there was a strong underlying contextually-driven (i.e., through felt stigma) effect of distress on the study outcomes. Put another way, had the moderating effect of felt stigma not been accounted for in our analysis, the underlying associations between psychological distress and each of the outcomes would have been obscured, demonstrating the importance of careful analysis of minority stress interactions when examining correlates of psychological distress and HIV screening and prevention engagement.

Our results show that psychological distress alone was not associated with HIV screening and prevention engagement, but when felt stigma was high-that is, when one expects rejection and discrimination in their interactions with the world - psychological distress was associated with reduced engagement in HIV screening and prevention efforts. Although we cannot study the psychological processes involved, it is plausible that fear and hypervigilance related to one's sexual identity may lead to avoidance of preventive behaviors (e.g., testing for HIV). Research has shown that sexual minority men who feel stigmatized due to their sexual orientation, as well as those who wish to avoid the stigma and discrimination associated with becoming HIV positive, may avoid testing for HIV altogether. ${ }^{45}$ Indeed, in a study of rural gay men, awareness of anti-gay bias in the community was related to unwillingness to request an HIV test from their personal physician due to fear of being perceived as gay, despite understanding HIV risk. ${ }^{46}$ This explanation is consistent with our finding that among men who felt little stigma, increased psychological distress was associated with greater adherence to HIV preventive behaviors. Although more research is needed to better understand this finding, it may highlight that in low-stigma settings, sexual minority men are more comfortable seeking care for both mental and sexual health needs. ${ }^{47}$

\section{HIV prevention among diverse MSM populations}

Key sociodemographic differences were also found with regard to HIV screening and prevention. Although men from all age cohorts were equally likely to have tested for $\mathrm{HIV}$ in the past year and were equally familiar with PrEP, 


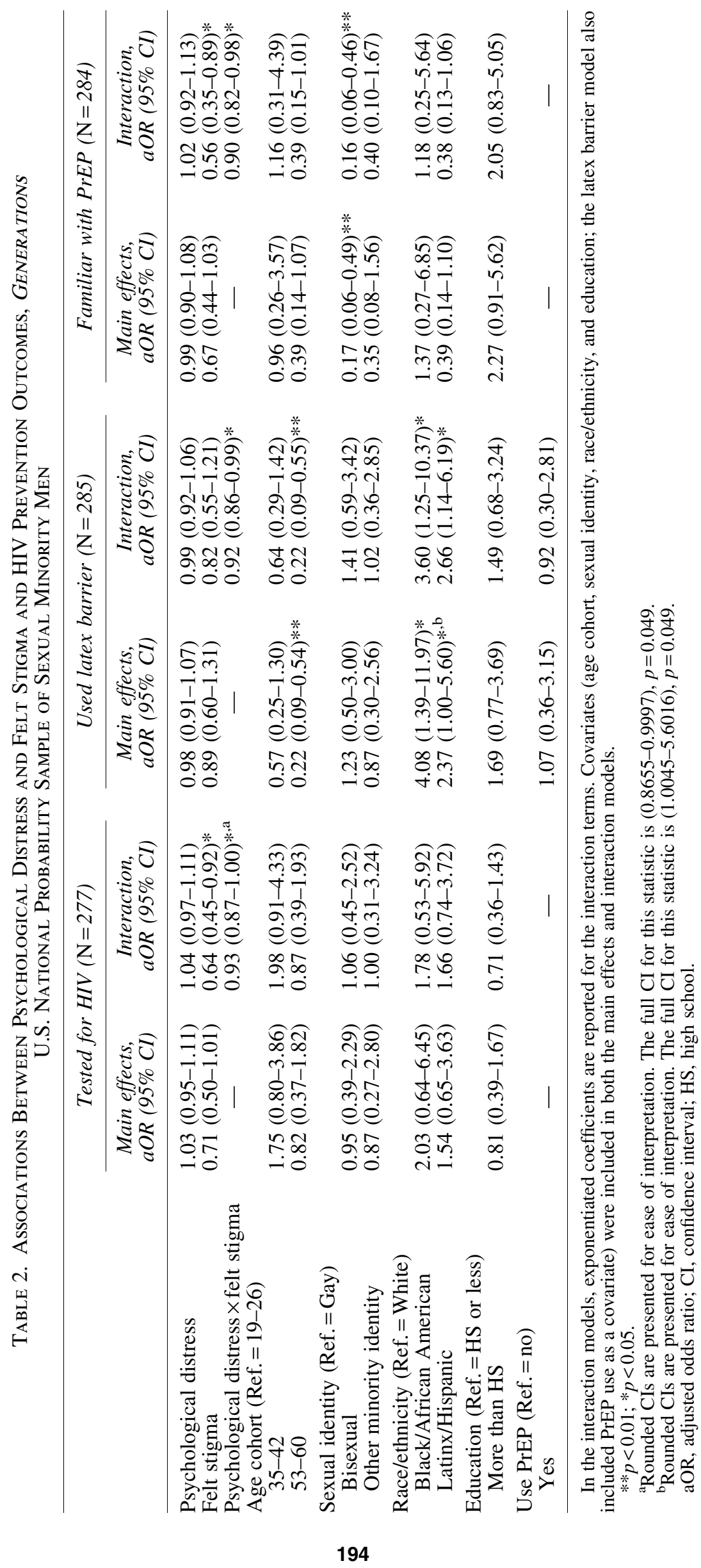



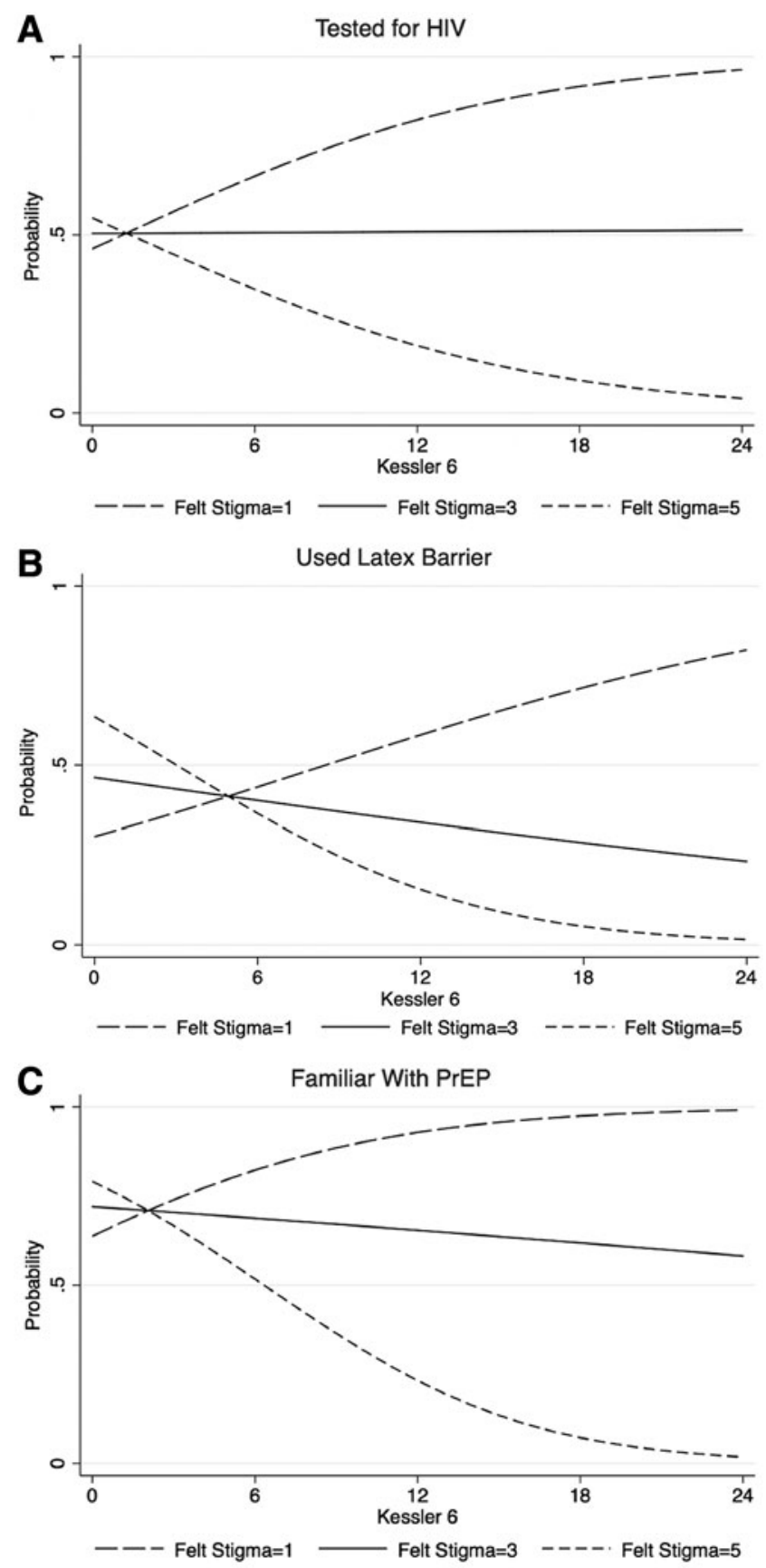

FIG. 1. Predicted probabilities of (A) HIV testing, (B) use of latex barriers, and $(\mathbf{C})$ familiarity with PrEP by psychological distress and felt stigma. PrEP, pre-exposure prophylaxis.

older men had significantly lower odds of using latex barriers compared to men in the youngest age cohort. Furthermore, compared to White men, Black and Latinx men had increased odds of using latex barriers. Although our sample included only 21 Black respondents (and hence, odds ratios with wide CIs), these findings are consistent with some prior research. ${ }^{48,49}$ Finally, compared to gay men, bisexual men had lower odds of being familiar with PrEP. Together, these findings may reflect the concerted effort that has been made to reduce HIV incidence among young men of color, who continue to have a higher rate of transmission than
White men. ${ }^{50,51}$ However, these findings may also highlight gaps in HIV prevention campaigns, which cannot afford to ignore older or bisexual-identified men. To meaningfully reduce the burden of HIV, MSM of all ages and from diverse identity groups must be included. Furthermore, prevention efforts may potentially need to be tailored specifically to these groups.

\section{Policy and clinical implications}

In this study, felt stigma operated as a moderator of the association between psychological distress and HIV risk behavior; in communities with higher rates of perceived sexual minority stigma (i.e., less perceived acceptance of sexual minority people), those experiencing higher psychological distress were less engaged in HIV screening and prevention efforts. This highlights the importance of reducing stigma toward and rejection of sexual minority people and providing legal protections to prevent discriminatory and unfair treatment of sexual minority men in the communities where they live. Policies aimed at creating more tolerant and inclusive environments for sexual minority people may have positive indirect impacts on HIV screening and prevention efforts.

Clinicians should also be aware of the potential disparate impact of psychological distress on HIV screening and prevention. How their clients' mental health status interacts with the clients' perceptions about their communities may be more determinant of their HIV-related health behaviors than considering mental health status alone. These results suggest that psychoeducation about HIV screening and prevention should be incorporated into mental health practice with sexual minority men and especially in communities with high levels of stigma toward sexual minority people. Sexual health needs may be secondary to the specific targets of clinical work, but they are crucial for clients' overall health and well-being.

\section{Limitations}

The Generations study was not designed to comprehensively assess HIV screening and prevention behaviors among MSM; therefore, we were limited to the specific variables collected on the survey. For instance, although anal sex carries greater risk for HIV transmission than oral sex, ${ }^{52}$ our measure of sexual activity (self-defined) did not allow us to account for the type(s) of sex in which respondents engaged. Thus, our sample likely includes some respondents who are low risk for HIV transmission. In addition, we were able to assess whether respondents used latex barriers with sex partners, but not the frequencies or fidelities with which they used them, or for which types of sex (e.g., oral vs. anal) respondents used them. Future studies should consider a more comprehensive assessment of HIV risk and protective behaviors. In addition, while not a primary focus of this study, race/ethnicity was reduced to a single dimension for analysis, obscuring variability among respondents selecting one- versus multiple identities.

More work is needed to disentangle the complex psychosocial mechanisms underlying engagement in HIV screening and prevention. For instance, although the interaction between psychological distress and felt stigma indeed indicates that stigma provided a context in which psychological distress is negatively associated with HIV prevention behaviors, the interaction may not be unidirectional; increased psychological distress may also be expected to contribute to one 
perceiving high levels of stigma, regardless of actual community attitudes. Furthermore, although we did not find direct effects of either psychological distress or felt stigma on HIV screening and prevention engagement, from a psychological mediation perspective, it is plausible that psychological distress would mediate the association between felt stigma and engagement in HIV screening and prevention. ${ }^{20}$ Moreover, given that the interaction terms for the HIV testing and PrEP familiarity models were significant among the full sample, but not among nonusers of PrEP suggests that these interactions may be weak or were underpowered. Given the important implications of these findings for HIV prevention and care, these relationships warrant further study-ideally using larger samples. Finally, and related to these limitations, these data were cross-sectional. To better understand the causal associations among felt stigma, psychological distress, and HIV screening and prevention, additional research is needed, ideally using longitudinal study designs.

\section{Conclusions}

This study demonstrated, using a national probability sample of sexually active, HIV-negative, sexual minority men, that felt stigma modified the association of psychological distress with HIV screening and prevention engagement. In high-stigma contexts, increasing psychological distress was associated with lower probabilities of testing for HIV, use of latex barriers, and also familiarity with PrEP. These findings suggest the importance of considering social context when examining correlates of HIV screening and prevention and when formulating future HIV prevention interventions.

\section{Disclaimer}

The content of this article is solely the responsibility of the authors and does not necessarily represent the official views of the funding agencies, including the National Institutes of Health.

\section{Author Disclosure Statement}

No competing financial interests exist.

\section{Funding Information}

Research reported in this article is part of the Generations Study, supported by the Eunice Kennedy Shriver National Institute of Child Health and Human Development of the National Institutes of Health under Award No. R01HD078526. The Generations investigators are: Ilan H. Meyer (Principal Investigator), David M. Frost, Phillip L. Hammack, Marguerita Lightfoot, Stephen T. Russell, and Bianca D.M. Wilson (Co-Investigators, listed alphabetically). Support was also obtained from the UCLA Center for HIV Identification, Prevention, and Treatment Services (CHIPTS) under Award No. P30MH058107.

\section{References}

1. Purcell DW, Johnson CH, Lansky A, et al.: Estimating the population size of men who have sex with men in the United States to obtain HIV and syphilis rates. Open AIDS J 2012;6:98-107.

2. Centers for Disease Control and Prevention: HIV: Gay and bisexual men. 2019. Available at www.cdc.gov/hiv/group/ msm/index.html Accessed April 1, 2020.
3. Centers for Disease Control and Prevention: HIV testing. 2019. Available at www.cdc.gov/hiv/testing/index.html Accessed April 1, 2020.

4. Centers for Disease Control and Prevention: Condoms and STDs: Fact sheet for public health personnel. 2013. Available at www.cdc.gov/condomeffectiveness/latex.html. Accessed March 6, 2020.

5. Centers for Disease Control and Prevention: 2015 Sexually Transmitted Diseases Treatment Guidelines: Clinical prevention guidance. 2015. Available at www.cdc.gov/std/ tg2015/clinical.htm Accessed May 10, 2019.

6. Centers for Disease Control and Prevention: Pre-exposure prophylaxis (PrEP) for HIV prevention. 2014. Available at www.cdc.gov/nchhstp/newsroom/docs/factsheets/prep-fact sheet-508.pdf Accessed May 10, 2019.

7. Hammack PL, Meyer IH, Krueger EA, et al.: HIV testing and pre-exposure prophylaxis (PrEP) use, familiarity, and attitudes among gay and bisexual men in the United States: A national probability sample of three birth cohorts. PLoS One 2018;13:e0202806.

8. Klevens RM, Martin BM, Doherty R, et al.: Factors associated with pre-exposure prophylaxis in a highly insured population of urban men who have sex with men, 2014. AIDS Behav 2018;22:1201-1208.

9. Lachowsky NJ, Lin SY, Hull MW, et al.: Pre-exposure prophylaxis awareness among gay and other men who have sex with men in Vancouver, British Columbia, Canada. AIDS Behav 2016;20:1408-1422.

10. Parsons JT, Rendina HJ, Lassiter JM, et al.: Uptake of HIV preexposure prophylaxis (PrEP) in a national cohort of gay and bisexual men in the United States: The Motivational PrEP Cascade. J Acquir Immune Defic Syndr 2017;74:285-292.

11. Busby KK, Lytle S, Sajatovic M: Mental health comorbidity and HIV/AIDS. In: Mental Health Practitioner's Guide to HIV/AIDS. Edited by Loue S. New York: Springer Science+Business Media, 2013, pp 9-35.

12. Mangurian C, Cournos F, Schillinger D, et al.: Low rates of HIV testing among adults with severe mental illness receiving care in community mental health settings. Psychiatr Serv 2017;68:443-448.

13. Batchelder AW, Safren S, Mitchell AD, et al.: Mental health in 2020 for men who have sex with men in the United States. Sex Health 2017;14:59-71.

14. King M, Semlyen J, Tai SS, et al.: A systematic review of mental disorder, suicide, and deliberate self harm in lesbian, gay and bisexual people. BMC Psychiatry 2008;8:70.

15. Cochran SD, Mays VM: Burden of psychiatric morbidity among lesbian, gay, and bisexual individuals in the California Quality of Life Survey. J Abnorm Psychol 2009;118:647-658.

16. Krueger EA, Meyer IH, Upchurch DM: Sexual orientation group differences in perceived stress and depressive symptoms among young adults in the United States. LGBT Health 2018;5:242-249.

17. Hendricks ML, Testa RJ: A conceptual framework for clinical work with transgender and gender nonconforming clients: An adaptation of the Minority Stress Model. Prof Psychol Res Pract 2012;43:460-467.

18. Hatzenbuehler ML, Nolen-Hoeksema S, Erickson SJ: Minority stress predictors of HIV risk behavior, substance use, and depressive symptoms: Results from a prospective study of bereaved gay men. Health Psychol 2008;27:455-462.

19. Wight RG, LeBlanc AJ, de Vries B, Detels R: Stress and mental health among midlife and older gay-identified men. Am J Public Health 2012;102:503-510. 
20. Hatzenbuehler ML: How does sexual minority stigma "get under the skin?" A psychological mediation framework. Psychol Bull 2009;135:707-730.

21. Fingerhut AW, Peplau LA, Gable SL: Identity, minority stress and psychological well-being among gay men and lesbians. Psychol Sex 2010;1:101-114.

22. Juster RP, Smith NG, Ouellet É, et al.: Sexual orientation and disclosure in relation to psychiatric symptoms, diurnal cortisol, and allostatic load. Psychosom Med 2013;75: 103-116.

23. Lewis RJ, Derlega VJ, Griffin JL, Krowinski AC: Stressors for gay men and lesbians: Life stress, gay-related stress, stigma consciousness, and depressive symptoms. J Soc Clin Psychol 2003;22:716-729.

24. Goldbach JT, Tanner-Smith EE, Bagwell M, Dunlap S: Minority stress and substance use in sexual minority adolescents: A meta-analysis. Prev Sci 2014;15:350-363.

25. McCabe SE, Bostwick WB, Hughes TL, et al.: The relationship between discrimination and substance use disorders among lesbian, gay, and bisexual adults in the United States. Am J Public Health 2010;100:1946-1952.

26. Safren SA, Reisner SL, Herrick A, et al.: Mental health and HIV risk in men who have sex with men. J Acquir Immune Defic Syndr 2010;55:S74-S77.

27. Frost DM, Parsons JT, Nanin JE: Stigma, concealment and symptoms of depression as explanations for sexually transmitted infections among gay men. J Health Psychol 2007; 12:636-640.

28. Rendina HJ, Gamarel KE, Pachankis JE, et al.: Extending the minority stress model to incorporate HIV-positive gay and bisexual men's experiences: A longitudinal examination of mental health and sexual risk behavior. Ann Behav Med 2017;51:147-158.

29. U.S. Department of Health \& Human Services. What is 'ending the HIV epidemic: A plan for America'? 2019. Available at www.hiv.gov/federal-response/ending-thehiv-epidemic/overview Accessed May 10, 2019.

30. Hatzenbuehler ML, Phelan JC, Link BG: Stigma as a fundamental cause of population health inequalities. Am J Public Health 2013;103:813-821.

31. Hatzenbuehler ML: The social environment and suicide attempts in lesbian, gay, and bisexual youth. Pediatrics 2011; 127:896-903.

32. Meyer IH: Prejudice, social stress, and mental health in lesbian, gay, and bisexual populations: Conceptual issues and research evidence. Psychol Bull 2003;129:674-697.

33. Herek GM: Hate crimes and stigma-related experiences among sexual minority adults in the United States: Prevalence estimates from a national probability sample. J Interpers Violence 2009;24:54-74.

34. Herek GM, Saha S, Burack J: Stigma and psychological distress in people with HIV/AIDS. Basic Appl Soc Psychol 2013;35:41-54.

35. Oldenburg CE, Perez-Brumer AG, Hatzenbuehler ML, et al.: State-level structural sexual stigma and HIV prevention in a national online sample of HIV-uninfected men who have sex with men in the United States. AIDS 2015;29:837845.

36. Lapinski MK, Braz ME, Maloney EK: The down low, social stigma, and risky sexual behaviors: Insights from AfricanAmerican men who have sex with men. J Homosex 2010; 57:610-633.

37. Arnold EA, Rebchook GM, Kegeles SM: 'Triply cursed': Racism, homophobia and HIV-related stigma are barriers to regu- lar HIV testing, treatment adherence and disclosure among young black gay men. Cult Health Sex 2014;16:710-722.

38. Golub SA, Gamarel KE: The impact of anticipated HIV stigma on delays in HIV testing behaviors: Findings from a community-based sample of men who have sex with men and transgender women in New York City. AIDS Patient Care STDS 2013;27:621-627.

39. Diaz RM, Ayala G, Bein E, et al.: The impact of homophobia, poverty, and racism on the mental health of gay and bisexual Latino men: Findings from 3 US cities. Am J Public Health 2001;91:927-932.

40. Koblin BA, Husnik MJ, Colfax G, et al.: Risk factors for HIV infection among men who have sex with men. AIDS 2006;20:731-739.

41. Krueger EA, Lin A, Kittle KR, Meyer IH: Generations quantitative survey methods v.16.; 2019. Available at www .generations-study.com/methods Accessed March 6, 2020.

42. Gallup. Methodology: How Does Gallup Daily Tracking Work? n.d. Available at www.gallup.com/174155/gallupdaily-tracking-methodology.aspx Accessed May 10, 2019.

43. Kelley CF, Kahle E, Siegler A, et al.: Applying a PrEP continuum of care for men who have sex with men in Atlanta, Georgia. Clin Infect Dis 2015;61:1590-1597.

44. National Comorbidity Survey: Kessler 6-Self Report Q1 (a)-(f). Available at www.integration.samhsa.gov/images/ res/K6 Questions.pdf Accessed May 10, 2019.

45. Smit PJ, Brady M, Carter M, et al.: HIV-related stigma within communities of gay men: A literature review. AIDS Care 2012;24:405-412.

46. Williams ML, Bowen AM, Horvath KJ: The social/sexual environment of gay men residing in a rural frontier state: Implications for the development of HIV prevention programs. J Rural Health 2005;21:48-55.

47. Pachankis JE, Hatzenbuehler ML, Hickson F, et al.: Hidden from health: Structural stigma, sexual orientation concealment, and HIV across 38 countries in the European MSM Internet Survey. AIDS 2015;29:1239-1246.

48. Maulsby C, Millett G, Lindsey K, et al.: HIV among black men who have sex with men (MSM) in the United States: A review of the literature. AIDS Behav 2014;18:10-25.

49. Magnus M, Kuo I, Phillips G 2nd, et al.: Elevated HIV prevalence despite lower rates of sexual risk behaviors among black men in the District of Columbia who have sex with men. AIDS Patient Care STDS 2010;24:615-622.

50. Centers for Disease Control and Prevention: HIV: African Americans. 2019. Available at www.cdc.gov/hiv/group/racial ethnic/africanamericans/index.html Accessed May 10, 2019.

51. Centers for Disease Control and Prevention: HIV and Hispanics/Latinos. 2019. Available at www.cdc.gov/hiv/ group/racialethnic/hispaniclatinos/index.html Accessed May 10, 2019.

52. Mastro TD, de Vincenzi I: Probabilities of sexual HIV-1 transmission. AIDS 1996;10:S75-S82.

Address correspondence to: Evan A. Krueger, PhD, MPH, MSW Department of Preventive Medicine Keck School of Medicine University of Southern California 2001 North Soto Street, 302-04 Los Angeles, CA 90032 USA

E-mail: eakruege@usc.edu 